Bull. Korean Math. Soc. 46 (2009), No. 4, pp. 657-671

DOI 10.4134/BKMS.2009.46.4.657

\title{
A STRUCTURE THEOREM FOR COMPLETE INTERSECTIONS
}

\author{
Eun Jeong Choi, Oh-Jin Kang, and Hyoung J. Ko
}

\begin{abstract}
Buchsbaum and Eisenbud proved a structure theorem for Gorenstein ideals of grade 3. In this paper we derive a class of the perfect ideals from a class of the complete matrices. From this we give a structure theorem for complete intersections of grade $g>3$.
\end{abstract}

\section{Introduction}

Let $R$ be a Noetherian local ring and $I$ a perfect ideal of grade $g$ in $R$. Many people have been studying the algebra structure on the minimal free resolution of $R / I$, in particular, Gorenstein ideals, the ideals of type 1. In 1968, Burch [3] characterized perfect ideals of grade 2 by showing a structure theorem due to Hilbert in a special case: every perfect ideal of grade 2 generated by $n$ elements is the ideal of $(n-1)$ st order minors of an $(n-1) \times n$ matrix. In 1977, Buchsbaum and Eisenbud [2] gave a structure theorem for Gorenstein ideals of grade 3 which says that every Gorenstein ideal of grade 3 in $R$ is generated by the maximal order Pfaffians of an alternating matrix. However a structure theorem for Gorenstein ideals of grade 4 is more complicated than that of grade 3 and not completely known. In 1987, Brown [1] described a structure theorem for a certain class of perfect ideals $I$ which have grade 3 , type 2 and $\lambda(I)=\operatorname{dim}_{k} \Lambda_{1}^{2}>0$, where $\lambda(I)$ is a numerical invariant defined in [5]. In 1989, Sanchez [7] gave a structure theorem for type 3, grade 3 perfect ideals which have $\lambda(I)=\operatorname{dim}_{k} \Lambda_{1}^{2}=2$ or greater. In this paper we will describe a structure theorem for complete intersections of grade $g>3$, which says that every complete intersection of grade $g>3$ in $R$ is generated by the elements $x_{i}$ 's, where $x_{i}^{g-1}$ is the determinant of the $(g-1) \times(g-1)$ diagonal matrix drawn from a complete matrix of grade $g$ for each $i(1 \leq i \leq g)$.

In Section 2 we review some of the properties of alternating matrices, linkage theory, and a structure theorem for Gorenstein ideals of grade 3.

In Section 3 we give the concept of a complete matrix of grade 4 and provide a structure theorem for complete intersections of grade 4 .

Received August 2, 2008.

2000 Mathematics Subject Classification. 13C05, 13C14, 13C40, 14M10.

Key words and phrases. complete intersection of grade $g$, structure theorem. 
In Section 4 we introduce a complete matrix $f$ of grade $g>3$, and define the ideal $\mathcal{K}_{g-1}(f)$ associated with $f$. Then we prove a structure theorem for complete intersections of grade $g>3$. The structure theorem [4] for complete intersections of grade 4 is just a special case of our main Theorem 4.10. Throughout this paper, we assume that all rings are a Noetherian local ring with maximal ideal $\mathfrak{m}$ unless otherwise stated.

\section{Gorenstein ideals of grade 3}

The grade of a proper ideal $I$ in $R$ is the length of the maximal $R$-sequence contained in $I$. We say that an ideal $I$ of grade $g$ is perfect if grade $I=$ $\operatorname{prodim}_{R}(R / I)=g$. If $I$ is a perfect ideal of grade $g$, then the type of $I$ is defined to be the dimension of the $R / \mathfrak{m}$-vector space $\operatorname{Ext}_{R}^{g}(R / \mathfrak{m}, R / I)$. A perfect ideal $I$ of grade $g$ is Gorenstein if type $I=1$, equivalently, if $\mathbb{F}$ is the minimal free resolution of $R / I$,

$$
\mathbb{F}: 0 \longrightarrow F_{g} \stackrel{\varphi_{g}}{\longrightarrow} F_{g-1} \stackrel{\varphi_{g-1}}{\longrightarrow} \cdots \stackrel{\varphi_{2}}{\longrightarrow} F_{1} \stackrel{\varphi_{1}}{\longrightarrow} F_{0}(=R),
$$

then the rank of $F_{g}$ is 1 . A perfect ideal $I$ of grade $g$ is a complete intersection if it is generated by $g$ elements, and is an almost complete intersection if it is minimally generated by $g+1$ elements.

Let $R$ be a commutative ring, and $F$ a finite free $R$-module. An $R$-module homomorphism $\varphi: F \rightarrow F^{*}$ is said to be alternating if with respect to some (and therefore any) basis of $F$ and the corresponding dual basis of $F^{*}$, the matrix $\varphi$ is alternating, i.e., skew-symmetric and all its diagonal entries are 0 . Now suppose that $\varphi$ is alternating, choose a basis of $F$ and the corresponding dual basis of this, and identify $\varphi$ with the corresponding matrix $\left(\varphi_{i j}\right)$. If $\operatorname{rank} F$ is odd, then $\operatorname{det} \varphi=0$, and if $\operatorname{rank} F$ is even, then there exists an element $\operatorname{Pf}(\varphi) \in R$, called the Pfaffian of $\varphi$, which is a polynomial function of the entries of $\varphi$, such that $\operatorname{det} \varphi=\operatorname{Pf}(\varphi)^{2}$. We set $\operatorname{Pf}(\varphi)=0$ if $\operatorname{rank} F$ is odd. Pfaffians can be developed along a row just like the determinants. Denote by $\operatorname{Pf}_{r}(\varphi)$ the ideal generated by the $r$ th order Pfaffians of $\varphi$. With these concepts Buchsbaum and Eisenbud gave a complete structure for Gorenstein ideals of grade 3:

Theorem 2.1 ([2]). Let $R$ be a Noetherian local ring with maximal ideal $\mathfrak{m}$.

(1) Let $F$ be a free $R$-module with rank $F=n$, where $n \geqslant 3$ is an odd integer. Let $\varphi: F^{*} \rightarrow F$ be an alternating map whose image is contained in $\mathfrak{m} F$. Suppose that $P f_{n-1}(\varphi)$ has grade 3 . Then $P f_{n-1}(\varphi)$ is a Gorenstein ideal minimally generated by $n$ elements.

(2) Every Gorenstein ideal of grade 3 arises as in (1).

Now we review some of the notions in the linkage theory formulated by Peskine and Szpiro in [6].

Definition 2.2. Let $I$ and $J$ be two ideals in a Gorenstein ring $R$ (not necessarily local). 
(1) If there exists an $R$-regular sequence $\boldsymbol{\alpha}=\alpha_{1}, \alpha_{2}, \ldots, \alpha_{g}$ in $I \cap J$ such that $J=(\boldsymbol{\alpha}): I$ and $I=(\boldsymbol{\alpha}): J$, then $I$ and $J$ are said to be linked (with respect to $\boldsymbol{\alpha})$.

(2) If $I$ and $J$ are linked and if $\operatorname{Ass}(R / I) \cap \operatorname{Ass}(R / J)=\emptyset$, equivalently, if $I$ and $J$ are linked (with respect to $\boldsymbol{\alpha}$ ) and if $I \cap J=(\boldsymbol{\alpha})$, then $I$ and $J$ are said to be geometrically linked.

Let $R$ be a Gorenstein local ring of Krull dimension $g$ with maximal ideal $\mathfrak{m}$. If $I$ and $J$ are perfect ideals of grade $g$, then they are not geometrically linked because $(R / I)$ and $(R / J)$ are both zero-dimensional artinian local rings. Peskine and Szpiro gave a method of constructing a Gorenstein ideal of grade $g+1$ from two perfect ideals of grade $g$ :

Theorem $2.3([6])$. Let $R$ be a Gorenstein local ring with maximal ideal $\mathfrak{m}$. Let $I$ and $J$ be geometrically linked Cohen-Macaulay ideals of grade $g$ by a regular sequence $\mathbf{x}=x_{1}, x_{2}, \ldots, x_{g}$ and let $K=I+J$. Then $K$ is a Gorenstein ideal of grade $g+1$.

Let $F$ be a free $R$-module with a basis $\left\{e_{1}, e_{2}, \ldots, e_{n}\right\}$ and let $I$ be an ideal generated by a regular sequence $\mathbf{x}=x_{1}, x_{2}, \ldots, x_{n}$. Let $\mathbb{K}(\mathbf{x})$ be the Koszul complex defined by $\mathbf{x}=x_{1}, x_{2}, \ldots, x_{n}$. Then

$$
\mathbb{K}(\mathbf{x}): 0 \longrightarrow \wedge^{n} F \stackrel{d_{n}}{\longrightarrow} \wedge^{n-1} F \stackrel{d_{n-1}}{\longrightarrow} \cdots \stackrel{d_{2}}{\longrightarrow} \wedge^{1} F \stackrel{d_{1}}{\longrightarrow} \wedge^{0} F
$$

is the minimal free resolution of $R / I$, where $d_{1}\left(e_{i}\right)=x_{i}$ for each $i$ with $1 \leqslant i \leqslant$ $n$, and for each $p$ with $1 \leqslant p \leqslant n, d_{p}: \wedge^{p} F \rightarrow \wedge^{p-1} F$ is given by

$$
d_{p}\left(e_{i_{1}} \wedge e_{i_{2}} \wedge \cdots \wedge e_{i_{p}}\right)=\sum_{j=1}^{p}(-1)^{j-1} d_{1}\left(e_{i_{j}}\right) e_{i_{1}} \wedge e_{i_{2}} \wedge \cdots \wedge \widehat{e}_{i_{j}} \wedge \cdots \wedge e_{i_{p}}
$$

For example, if $\mathbf{x}=x_{1}, x_{2}, x_{3}, x_{4}, x_{5}$ is a regular sequence on $R$, then $d_{2}$ has the form

$$
d_{2}=\left[\begin{array}{cccccccccc}
-x_{2} & -x_{3} & -x_{4} & -x_{5} & 0 & 0 & 0 & 0 & 0 & 0 \\
x_{1} & 0 & 0 & 0 & -x_{3} & -x_{4} & -x_{5} & 0 & 0 & 0 \\
0 & x_{1} & 0 & 0 & x_{2} & 0 & 0 & -x_{4} & -x_{5} & 0 \\
0 & 0 & x_{1} & 0 & 0 & x_{2} & 0 & x_{3} & 0 & -x_{5} \\
0 & 0 & 0 & x_{1} & 0 & 0 & x_{2} & 0 & x_{3} & x_{4}
\end{array}\right]
$$

The exterior algebra $\wedge F$ is a graded Hopf algebra such that $x \wedge y=(-1)^{p q} y \wedge$ $x$ for $x \in \wedge^{p} F$ and $y \in \wedge^{q} F$ and $x \wedge x=0$ for any homogeneous element $x$ of odd degree. It is well-known that the algebra structure on the Koszul complex which gives the minimal free resolution of a complete intersection is an exterior algebra. 


\section{Complete intersections of grade 4}

In this section we start with a skew-symmetrizable matrix, and a complete matrix of grade 4 which play important roles in describing the complete intersections of grade 4 .

Definition 3.1. Let $R$ be a commutative ring with identity. An $n \times n$ matrix $X=\left(x_{i j}\right)$ over $R$ is said to be generalized alternating or skew-symmetrizable if there exist nonzero $n \times n$ diagonal matrices $D^{\prime}=\operatorname{diag}\left(u_{1}, u_{2}, \ldots, u_{n}\right)$ and $D=\operatorname{diag}\left(w_{1}, w_{2}, \ldots, w_{n}\right)$ with entries in $R$ such that $D^{\prime} X D$ is alternating. We denote by $\operatorname{GA}_{n}(R)$ the set of all skew-symmetrizable $n \times n$ matrices over $R$. If there is no ambiguity about the $\operatorname{ring} R$, then $\mathrm{GA}_{n}(R)$ is denoted by $\mathrm{GA}_{n}$.

Notice that every alternating matrix is skew-symmetrizable. For an $n \times n$ skew-symmetrizable matrix $X$, we denote $\mathcal{A}(X)$ to be an alternating matrix $D^{\prime} X D$ for some diagonal matrices $D^{\prime}$ and $D$. To define a complete intersection of grade 4 , we need to describe the submatrices of the given matrix in detail. A $p \times q$ submatrix of an $m \times n$ matrix $f$ is a matrix obtained from $f$ by taking the $p q$ entries at the intersections of the $i_{1}$ th, $i_{2}$ th, $\ldots, i_{p}$ th rows and the $j_{1}$ th, $j_{2}$ th $, \ldots, j_{q}$ th columns of $f$, where $1 \leq i_{1}<i_{2}<\cdots<i_{p} \leq m$ and $1 \leq j_{1}<j_{2}<\cdots<j_{q} \leq n$. The corresponding $p \times q$ submatrix of $f$ is denoted by

$$
f\left(i_{1}, i_{2}, \ldots, i_{p} \mid j_{1}, j_{2}, \ldots, j_{q}\right) .
$$

Notice that the $p \times q$ matrix $f\left(i_{1}, i_{2}, \ldots, i_{p} \mid j_{1}, j_{2}, \ldots, j_{q}\right)$ consisting of the $p q$ entries at the intersection of these rows and columns of $f$ could not be a submatrix of $f$ unless $1 \leq i_{1}<i_{2}<\cdots<i_{p} \leq m$ and $1 \leq j_{1}<j_{2}<\cdots<j_{q} \leq n$. Next we get into the skew-symmetrizable matrices and the special properties of the second differential map $d_{2}$ of the Koszul complex $\mathbb{K}(\mathbf{x})$

$$
\mathbb{K}(\mathbf{x}): 0 \longrightarrow \wedge^{4} F \stackrel{d_{4}}{\longrightarrow} \wedge^{3} F \stackrel{d_{3}}{\longrightarrow} \wedge^{2} F \stackrel{d_{2}}{\longrightarrow} \wedge^{1} F \stackrel{d_{1}}{\longrightarrow} \wedge^{0} F
$$

defined by a regular sequence $\mathbf{x}=x_{1}, x_{2}, x_{3}, x_{4}$ on $R$. With respect to the standard basis of $F, d_{2}$ has the following form

$$
d_{2}=\left[\begin{array}{cccccc}
-x_{2} & -x_{3} & -x_{4} & 0 & 0 & 0 \\
x_{1} & 0 & 0 & -x_{3} & -x_{4} & 0 \\
0 & x_{1} & 0 & x_{2} & 0 & -x_{4} \\
0 & 0 & x_{1} & 0 & x_{2} & x_{3}
\end{array}\right]
$$

Proposition 3.2. With the notation as above, the second differential map $d_{2}$ of the Koszul complex satisfies the following properties:

(1) There are four disjoint pairs $(S, T)$ of two $4 \times 3$ submatrices of $d_{2}$;

(2) By removing a row and interchanging columns, each pair $(S, T)$ can be reduced to a pair $(\bar{S}, \bar{T})$ of $3 \times 3$ matrices such that $\bar{S}$ is a diagonal matrix whose determinant is the nonzero 3 rd power element $x^{3}$ for some $x \in R$, and $\bar{T}$ is a skew-symmetrizable matrix with grade $\operatorname{Pf}_{2}(\mathcal{A}(\bar{T}))=3$. 
Proof. Let $S_{1}=d_{2}(1,2,3,4 \mid 1,2,3)$ and $T_{1}=d_{2}(1,2,3,4 \mid 4,5,6)$ be the disjoint $4 \times 3$ submatrices of $d_{2}$. Then the submatrix obtained by removing the first row of $S_{1}$ is a $3 \times 3$ diagonal matrix $\bar{S}_{1}$ whose determinant is equal to $x_{1}^{3}$. Removing the first row and interchanging columns 1 and 3 of $T_{1}$, we have the $3 \times 3$ matrix $\bar{T}_{1}$. Then $\bar{T}_{1}$ is skew-symmetrizable, since it becomes an alternating matrix by multiplying the second column of it by -1 . Since $x_{2}, x_{3}, x_{4}$ is a regular sequence on $R, \operatorname{Pf}_{2}\left(\mathcal{A}\left(\bar{T}_{1}\right)\right)=\left(x_{2}, x_{3}, x_{4}\right)$ has grade 3 . Similarly, we can take the disjoint submatrices of $d_{2}$;

$$
\begin{aligned}
& S_{2}=d_{2}(1,2,3,4 \mid 1,4,5) \text { and } T_{2}=d_{2}(1,2,3,4 \mid 2,3,6), \\
& S_{3}=d_{2}(1,2,3,4 \mid 2,4,6) \text { and } T_{3}=d_{2}(1,2,3,4 \mid 1,3,5), \\
& S_{4}=d_{2}(1,2,3,4 \mid 3,5,6) \text { and } T_{4}=d_{2}(1,2,3,4 \mid 1,2,4) .
\end{aligned}
$$

The similar argument gives us the $3 \times 3$ diagonal matrix $\bar{S}_{i}$ whose determinant is equal to $x_{i}^{3}$ or $\left(-x_{i}\right)^{3}$, and the $3 \times 3$ skew-symmetrizable matrix $\bar{T}_{i}$ with grade $\mathrm{Pf}_{2}\left(\mathcal{A}\left(\bar{T}_{i}\right)\right)=3$ for $i=2,3,4$.

Definition 3.3. Let $R$ be a commutative ring with identity. A $4 \times 6$ matrix $f$ over $R$ is said to be a complete matrix of grade 4 if

(1) $f$ has four distinct pairs $(S, T)$ of disjoint $4 \times 3$ submatrices;

(2) By removing a row and interchanging columns, each pair $(S, T)$ is reduced to a pair $(\bar{S}, \bar{T})$ of $3 \times 3$ matrices such that $\bar{S}$ is a diagonal matrix whose determinant is a nonzero 3rd power element $x^{3}$ for some $x \in R$, and $\bar{T}$ is a skew-symmetrizable matrix with grade $\operatorname{Pf}_{2}(\mathcal{A}(\bar{T}))=3$.

The following example illustrates Definition 3.3.

Example 3.4. Let $x, y, z$, and $w$ be a regular sequence on a commutative ring $R$. Let $f$ be a $4 \times 6$ matrix given by

$$
f=\left[\begin{array}{cccccc}
0 & 0 & -y & -w & -z & 0 \\
0 & -z & x & 0 & 0 & -w \\
-w & y & 0 & 0 & x & 0 \\
z & 0 & 0 & x & 0 & y
\end{array}\right]
$$

Then $f$ is a complete matrix of grade 4 . To see this, we find four distinct pairs of disjoint $4 \times 3$ submatrices $S_{i}$ and $T_{i}$ of $f$ satisfying the properties in Proposition 3.2. First we consider two submatrices of $f$;

$$
S_{1}=\left[\begin{array}{ccc}
-y & -w & -z \\
x & 0 & 0 \\
0 & 0 & x \\
0 & x & 0
\end{array}\right] \quad \text { and } \quad T_{1}=\left[\begin{array}{ccc}
0 & 0 & 0 \\
0 & -z & -w \\
-w & y & 0 \\
z & 0 & y
\end{array}\right] \text {, }
$$

that is, $S_{1}=f(1,2,3,4 \mid 3,4,5)$ and $T_{1}=f(1,2,3,4 \mid 1,2,6)$. So $S_{1}$ and $T_{1}$ are disjoint. By removing the first row and interchanging the second and the third columns of $S_{1}$ and $T_{1}$, we can get the $3 \times 3$ matrices $\bar{S}_{1}=S_{1}(2,3,4 \mid 1,3,2)$ and $\bar{T}_{1}=T_{1}(2,3,4 \mid 1,3,2)$. Then $\bar{S}_{1}$ is a diagonal matrix whose determinant is a nonzero element $x^{3}$ and $\bar{T}_{1}$ is skew-symmetrizable since $\bar{T}_{1} \operatorname{diag}(1,-1,1)$ 
is alternating. It is easy to show that $\operatorname{Pf}_{2}\left(\mathcal{A}\left(\bar{T}_{1}\right)\right)$ has grade 3 . Similarly, we consider the submatrices of $f$;

$$
\begin{aligned}
& S_{2}=f(1,2,3,4 \mid 2,3,6) \text { and } T_{2}=f(1,2,3,4 \mid 1,4,5), \\
& S_{3}=f(1,2,3,4 \mid 1,2,5) \text { and } T_{3}=f(1,2,3,4 \mid 3,4,6), \\
& S_{4}=f(1,2,3,4 \mid 1,4,6) \text { and } T_{4}=f(1,2,3,4 \mid 2,3,5) .
\end{aligned}
$$

Clearly, $4 \times 3$ submatrices $S_{i}$ and $T_{i}$ of $f$ are disjoint for $i=2,3,4$. The similar argument gives us the following $3 \times 3$ matrices;

$$
\begin{aligned}
& \bar{S}_{2}=S_{2}(1,3,4 \mid 2,1,3) \text { and } \bar{T}_{2}=T_{2}(1,3,4 \mid 1,2,3), \\
& \bar{S}_{3}=S_{3}(1,2,4 \mid 3,2,1) \text { and } \bar{T}_{3}=T_{3}(1,2,4 \mid 3,2,1), \\
& \bar{S}_{4}=S_{4}(1,2,3 \mid 2,3,1) \text { and } \bar{T}_{4}=T_{4}(1,2,3 \mid 1,3,2) .
\end{aligned}
$$

And $\operatorname{det} \bar{S}_{2}=(-y)^{3}$, $\operatorname{det} \bar{S}_{3}=z^{3}$ and $\operatorname{det} \bar{S}_{4}=(-w)^{3}$ are nonzero 3rd power elements and

$$
\begin{aligned}
& \operatorname{Pf}_{2}\left(\mathcal{A}\left(\bar{T}_{1}\right)\right)=(y, z, w), \quad \operatorname{Pf}_{2}\left(\mathcal{A}\left(\bar{T}_{2}\right)\right)=(x, z, w), \\
& \operatorname{Pf}_{2}\left(\mathcal{A}\left(\bar{T}_{3}\right)\right)=(x, y, w), \quad \operatorname{Pf}_{2}\left(\mathcal{A}\left(\bar{T}_{4}\right)\right)=(x, y, z) .
\end{aligned}
$$

Since $x, y, z, w$ is a regular sequence on $R$, these four ideals have all grade 3 . Hence the properties in Proposition 3.2 are satisfied.

We notice that if $f$ is a complete matrix of grade 4 , then the matrix obtained from $f$ by interchanging rows of $f$ also becomes a complete matrix of grade 4 .

Theorem 3.5 ([4]). Let $f=\left(f_{i j}\right)$ be $a 4 \times 6$ complete matrix of grade 4 .

(1) Every column of $f$ has exactly two nonzero entries.

(2) The number of nonzero rows in each $4 \times 2$ submatrix of $f$ is greater than 2.

(3) Each pair $(\bar{S}, \bar{T})$ of $3 \times 3$ matrices given in Definition 3.3 is uniquely determined.

Now we will define an ideal $\mathcal{K}_{3}(f)$ generated by the radical roots of the determinants of the $3 \times 3$ diagonal matrices $\bar{S}$ derived from a given complete matrix $f$ of grade 4 in Theorem 3.5.

Definition 3.6. Let $f$ be a $4 \times 6$ complete matrix of grade 4 . Let $\bar{S}_{i}$ be a unique $3 \times 3$ diagonal matrix reduced from the disjoint pair $\left(S_{i}, T_{i}\right)$ of $f$ such that $\operatorname{det} \bar{S}_{i}=x_{i}^{3}$ is nonzero for $i=1,2,3,4$. We define $\mathcal{K}_{3}(f)$ to be the ideal generated by the $x_{i}$ 's, that is,

$$
\mathcal{K}_{3}(f)=\left(x_{1}, x_{2}, x_{3}, x_{4}\right) .
$$

Next let us show that the ideal $\mathcal{K}_{3}(f)$ defines a complete intersection of grade 4 . Let $f$ be a complete matrix of grade 4 . By Theorem 3.5 we may 
assume

$$
f=\left[\begin{array}{cccccc}
f_{11} & f_{12} & f_{13} & 0 & 0 & 0 \\
f_{21} & 0 & 0 & f_{24} & f_{25} & 0 \\
0 & f_{32} & 0 & f_{34} & 0 & f_{36} \\
0 & 0 & f_{43} & 0 & f_{45} & f_{46}
\end{array}\right]
$$

Then we have

$$
\begin{aligned}
& \bar{S}_{1}=f(2,3,4 \mid 1,2,3) \text { and } \bar{T}_{1}=f(2,3,4 \mid 6,5,4), \\
& \bar{S}_{2}=f(1,3,4 \mid 1,4,5) \text { and } \bar{T}_{2}=f(1,3,4 \mid 6,3,2), \\
& \bar{S}_{3}=f(1,2,4 \mid 2,4,6) \text { and } \bar{T}_{3}=f(1,2,4 \mid 5,3,1), \\
& \bar{S}_{4}=f(1,2,3 \mid 3,5,6) \text { and } \bar{T}_{4}=f(1,2,3 \mid 4,2,1),
\end{aligned}
$$

i.e.,

$$
\begin{aligned}
\bar{S}_{1}=\left[\begin{array}{ccc}
f_{21} & 0 & 0 \\
0 & f_{32} & 0 \\
0 & 0 & f_{43}
\end{array}\right] \text { and } & \bar{T}_{1}=\left[\begin{array}{ccc}
0 & f_{25} & f_{24} \\
f_{36} & 0 & f_{34} \\
f_{46} & f_{45} & 0
\end{array}\right], \\
\bar{S}_{2}=\left[\begin{array}{ccc}
f_{11} & 0 & 0 \\
0 & f_{34} & 0 \\
0 & 0 & f_{45}
\end{array}\right] \text { and } & \bar{T}_{2}=\left[\begin{array}{ccc}
0 & f_{13} & f_{12} \\
f_{36} & 0 & f_{32} \\
f_{46} & f_{43} & 0
\end{array}\right], \\
\bar{S}_{3}=\left[\begin{array}{ccc}
f_{12} & 0 & 0 \\
0 & f_{24} & 0 \\
0 & 0 & f_{46}
\end{array}\right] \text { and } & \bar{T}_{3}=\left[\begin{array}{ccc}
0 & f_{13} & f_{11} \\
f_{25} & 0 & f_{21} \\
f_{45} & f_{43} & 0
\end{array}\right], \\
\bar{S}_{4}=\left[\begin{array}{ccc}
f_{13} & 0 & 0 \\
0 & f_{25} & 0 \\
0 & 0 & f_{36}
\end{array}\right] \text { and } & \bar{T}_{4}=\left[\begin{array}{ccc}
0 & f_{12} & f_{11} \\
f_{24} & 0 & f_{21} \\
f_{34} & f_{32} & 0
\end{array}\right] .
\end{aligned}
$$

Since $\bar{T}_{i} \operatorname{diag}\left(u_{i_{1}}, u_{i_{2}}, u_{i_{3}}\right)$ is alternating where $u_{i_{k}} \in\{ \pm 1\}$, we have the following identities

$$
\begin{aligned}
& f_{24}=f_{46} \text { or }-f_{46}, f_{25}=f_{36} \text { or }-f_{36}, f_{34}=f_{45} \text { or }-f_{45}, \\
& f_{12}=f_{46} \text { or }-f_{46}, f_{13}=f_{36} \text { or }-f_{36}, f_{32}=f_{43} \text { or }-f_{43}, \\
& f_{11}=f_{45} \text { or }-f_{45}, f_{13}=f_{25} \text { or }-f_{25}, f_{21}=f_{43} \text { or }-f_{43}, \\
& f_{11}=f_{34} \text { or }-f_{34}, f_{12}=f_{24} \text { or }-f_{24}, f_{21}=f_{32} \text { or }-f_{32} .
\end{aligned}
$$

Thus (3.2) and (3.3) give us

$$
\begin{aligned}
& \operatorname{det} \bar{S}_{1}=f_{21} f_{32} f_{43}=f_{21}^{3} \text { or }-f_{21}^{3}, \quad \operatorname{det} \bar{S}_{2}=f_{11} f_{34} f_{45}=f_{11}^{3} \text { or }-f_{11}^{3}, \\
& \operatorname{det} \bar{S}_{3}=f_{12} f_{24} f_{46}=f_{12}^{3} \text { or }-f_{12}^{3}, \operatorname{det} \bar{S}_{4}=f_{13} f_{25} f_{36}=f_{13}^{3} \text { or }-f_{13}^{3},
\end{aligned}
$$

and

$$
\begin{aligned}
& \mathrm{Pf}_{2}\left(\mathcal{A}\left(\bar{T}_{1}\right)\right)=\left(f_{11}, f_{12}, f_{13}\right), \mathrm{Pf}_{2}\left(\mathcal{A}\left(\bar{T}_{2}\right)\right)=\left(f_{21}, f_{13}, f_{12}\right), \\
& \operatorname{Pf}_{2}\left(\mathcal{A}\left(\bar{T}_{3}\right)\right)=\left(f_{21}, f_{13}, f_{11}\right), \mathrm{Pf}_{2}\left(\mathcal{A}\left(\bar{T}_{4}\right)\right)=\left(f_{21}, f_{12}, f_{11}\right) .
\end{aligned}
$$


Hence

$$
\operatorname{Pf}_{2}\left(\mathcal{A}\left(\bar{T}_{i}\right)\right) \subseteq \mathcal{K}_{3}(f)=\left(f_{21}, f_{11}, f_{12}, f_{13}\right) \text { for } i=1,2,3,4
$$

Thus we obtain the structure theorem for complete intersections of grade 4 .

Theorem 3.7 ([4]). Let $R$ be a Noetherian local ring with maximal ideal $\mathfrak{m}$.

(1) Let $F$ and $G$ be free $R$-modules with rank $F=6$ and rank $G=4$. Let $f=\left(f_{i j}\right): F \rightarrow G$ be a complete matrix of grade 4 such that $\operatorname{Im} f \subseteq \mathfrak{m} G$. With the notation as in Theorem 3.5, we assume that $\operatorname{Pf}_{2}\left(\mathcal{A}\left(\bar{T}_{i}\right)\right)+\operatorname{Pf}_{2}\left(\mathcal{A}\left(\bar{T}_{j}\right)\right)$ has grade 4 for some $i, j(i \neq j)$. Then the ideal $\mathcal{K}_{3}(f)$ is a complete intersection of grade 4 .

(2) Let $I=\left(x_{1}, x_{2}, x_{3}, x_{4}\right)$ be a complete intersection of grade 4 and let

$$
\mathbb{F}: 0 \longrightarrow R \stackrel{\varphi_{4}}{\longrightarrow} R^{4} \stackrel{\varphi_{3}}{\longrightarrow} R^{6} \stackrel{\varphi_{2}}{\longrightarrow} R^{4} \stackrel{\varphi_{1}}{\longrightarrow} R
$$

be the minimal free resolution of $R / I$. Then $\varphi_{2}$ and the transpose of $\varphi_{3}$ satisfy the part (1).

\section{Complete intersections of grade $g>4$}

In this section we construct the ideal $\mathcal{K}_{g}(f)$ associated with a complete matrix $f$ of grade $g>3$ and provide a structure theorem for complete intersections of grade $g>3$. We begin this section with easy lemmas.

Lemma 4.1. Let $R$ be a Noetherian local ring with maximal ideal $\mathfrak{m}$. For any positive integer $g>3$, let $\mathbf{x}=x_{1}, x_{2}, \ldots, x_{g}$ and $\mathbf{y}_{i}=x_{1}, x_{2}, \ldots, \widehat{x_{i}}, \ldots, x_{g}$ be regular sequences on $R$, where $\widehat{x}_{i}$ indicates that $x_{i}$ is to be omitted. Let $\mathbb{K}(\mathbf{x})$ and $\mathbb{K}\left(\mathbf{y}_{i}\right)$ be the Koszul complexes of $R /(\mathbf{x})$ and $R /\left(\mathbf{y}_{i}\right)$ for each $i=1,2,3, \ldots, g$. Let

$$
\mathbb{K}\left(x_{i}\right): 0 \longrightarrow R \stackrel{x_{i}}{\longrightarrow} R
$$

be a complex of free $R$-modules and $R$-maps. Then

(1) $\mathbb{K}(\mathbf{x}) \cong \mathbb{K}\left(x_{i}\right) \otimes \mathbb{K}\left(\mathbf{y}_{i}\right)$

(2) Let

$$
\mathbb{K}\left(\mathbf{y}_{i}\right): 0 \longrightarrow F_{g-1} \stackrel{\varphi_{i g-1}}{\longrightarrow} F_{g-2} \stackrel{\varphi_{i g-2}}{\longrightarrow} \cdots \stackrel{\varphi_{i 2}}{\longrightarrow} F_{1} \stackrel{\varphi_{i 1}}{\longrightarrow} R
$$

and

$$
\begin{aligned}
& \mathbb{K}\left(x_{i}\right) \otimes \mathbb{K}\left(\mathbf{y}_{i}\right): 0 \longrightarrow R \otimes F_{g-1} \stackrel{\phi_{i g}}{\longrightarrow} R \otimes F_{g-2} \oplus R \otimes F_{g-1} \stackrel{\phi_{i g-1}}{\longrightarrow} \\
& \cdots \stackrel{\phi_{i 2}}{\longrightarrow} R \otimes R \oplus R \otimes F_{1} \stackrel{\phi_{i 1}}{\longrightarrow} R \otimes R .
\end{aligned}
$$


Then we have

$$
\begin{aligned}
& \phi_{i 1}=\left[\begin{array}{ll}
x_{i} & \varphi_{i 1}
\end{array}\right], \quad \phi_{i k}=\left[\begin{array}{c|c}
(-1)^{k-1} \varphi_{i k-1} & 0 \\
x_{i} I & \varphi_{i k}
\end{array}\right] \text { for } k=2,3, \ldots, g-1, \\
& \phi_{i g}=\left[\begin{array}{c}
\varphi_{i g-1} \\
-x_{i}
\end{array}\right] .
\end{aligned}
$$

Proof. Clear.

Lemma 4.2. With the notation as above, let $t=\left(\begin{array}{l}g \\ 2\end{array}\right)$. Then, for each $i$

(1) Every column of $\phi_{i 2}$ has exactly two nonzero entries.

(2) The number of nonzero rows in each $g \times 2$ submatrix of $\phi_{i 2}$ is greater than 2 , that is, 3 or 4 .

Proof. This follows from the matrix form of $\phi_{i 2}$ (see (2.1) and (2.2)).

Now we can describe the special properties of $\phi_{i 2}$ in (4.1).

Proposition 4.3. With the notation as above and hypotheses:

(1) $\phi_{i 2}$ has $g$ disjoint pairs $\left(S_{k}, T_{k}\right)$ of a $g \times(g-1)$ submatrix $S_{k}$ and $a$ $g \times(t-g+1)$ submatrix $T_{k}$;

(2) By removing the ith row and interchanging columns of $\phi_{i 2}$, each pair $\left(S_{k}, T_{k}\right)$ can be reduced to a pair $\left(\bar{S}_{k}, \bar{T}_{k}\right)$, where $\bar{S}_{k}$ is a $(g-1) \times(g-1)$ diagonal matrix whose determinant is $x_{k}^{g-1}$, up to sign, and $\bar{T}_{k}$ is the second differential map in the Koszul complex $\mathbb{K}\left(\mathbf{y}_{k}\right)$.

Proof. (1) The first statement follows from the second statement.

(2) It is enough to prove the case $i=1$. For the sake of simplicity, $\phi_{12}$ can be written as the form

$$
\phi_{12}=\left[\begin{array}{c|c}
-\varphi_{11} & 0 \\
\hline x_{1} I & \varphi_{12}
\end{array}\right] .
$$

Let $S_{1}=\phi_{12}(1,2, \ldots, g \mid 1,2, \ldots, g-1)$ and $T_{1}=\phi_{12}(1,2, \ldots, g \mid g, g+$ $1, \ldots, t)$. Then clearly, $S_{1}$ and $T_{1}$ are disjoint. Taking $\bar{S}_{1}=x_{1} I$ and $\bar{T}_{1}=\varphi_{12}$ as submatrices of $\phi_{12}$, it is clear that $\operatorname{det} \bar{S}_{1}=\left(x_{1}\right)^{g-1}$ and $\bar{T}_{1}$ is the second differential map in the Koszul complex $\mathbb{K}\left(\mathbf{y}_{1}\right)$. Let $k>1$ be an integer with $2 \leq k \leq g$. It follows from Lemma 4.2 that every row of $\phi_{12}$ consists of exactly $g-1$ nonzero entries and exactly $t-g+1$ zero entries. Choose $S_{k}$ to be a $g \times(g-1)$ submatrix of $\phi_{12}$ such that all the entries of the $k$ th row are nonzero, and $T_{k}$ to be a $g \times(t-g+1)$ submatrix of $\phi_{12}$ such that all the entries of the $k$ th row are zero. Then clearly $S_{k}$ and $T_{k}$ are disjoint. Let $S_{k}^{\prime}$ and $T_{k}^{\prime}$ be the submatrices of $S_{k}$ and $T_{k}$ obtained by removing the $k$ th row of $S_{k}$ and $T_{k}$, respectively. By the part (1) of Lemma 4.2, every column of $S_{k}^{\prime}$ has exactly one 
nonzero entry. We observe from (4.2) that the nonzero entry in the lth column of $S_{k}^{\prime}$ is either $x_{k}$ or $-x_{k}$ for $l=1,2, \ldots, g-1$. The part (2) of Lemma 4.2 implies that every row of $S_{k}^{\prime}$ has exactly one nonzero entry. This implies that interchanging columns of $S_{k}^{\prime}$ produces a $(g-1) \times(g-1)$ diagonal matrix $\bar{S}_{k}$ whose main diagonal entries are either $x_{k}$ or $-x_{k}$. Thus $\operatorname{det} \bar{S}_{k}= \pm x_{k}^{g-1}$. It follows from the construction of $T_{k}^{\prime}$ and Lemma 4.2 that every column of $T_{k}^{\prime}$ has exactly two nonzero entries and the number of nonzero rows in each $(g-1) \times 2$ submatrix of $T_{k}^{\prime}$ is 3 . Since $T_{k}^{\prime}$ has $t-g+1=\left(\begin{array}{c}g-1 \\ 2\end{array}\right)$ columns and $g-1$ rows, interchanging columns of $T_{k}^{\prime}$ (if necessary) gives us the second differential map $\bar{T}_{k}$ in the Koszul complex $\mathbb{K}\left(\mathbf{y}_{k}\right)$ (see (4.2)). Actually, $\bar{T}_{k}$ has the form

$$
\bar{T}_{k}=\left[\begin{array}{l|l}
h_{k} & 0 \\
\hline d_{1} & h_{k}^{\prime}
\end{array}\right],
$$

where

$$
\begin{aligned}
& h_{k}=\left[\begin{array}{llllll}
-x_{2} & -x_{3} & \cdots & -\widehat{x_{k}} & \cdots & -x_{g}
\end{array}\right], \\
& d_{1}=\operatorname{diag}\left(x_{1}, x_{1}, \ldots, x_{1}\right) \text {, } \\
& h_{k}^{\prime}=\text { the second differential map in the Koszul complex } \mathbb{K}\left(\mathbf{y}_{1 k}\right) \text { for } \\
& \mathbf{y}_{1 k}=x_{2}, x_{3}, \ldots, \widehat{x_{k}}, \ldots, x_{g} .
\end{aligned}
$$

Thus we have the desired one $\bar{T}_{k}$.

To define the ideal $\mathcal{K}_{g-1}\left(\phi_{i 2}\right)$ associated with the map $\phi_{i 2}$ we need further properties of $\phi_{i 2}$.

Theorem 4.4. (1) With the notation as in Proposition 4.3 , for each $k(1 \leq k \leq$ g), a pair $\left(\bar{S}_{k}, \bar{T}_{k}\right)$ of matrices given in Proposition 4.3 is uniquely determined.

(2) If for each $k, \mathcal{K}_{g-2}\left(\bar{T}_{k}\right)$ is the ideal generated by the elements $x_{1}, x_{2}, \ldots$, $\widehat{x_{k}}, \ldots, x_{g}$ given in the proof of Proposition 4.3 , then $\mathcal{K}_{g-2}\left(\bar{T}_{k}\right)$ has grade $g-1$.

Proof. (1) This follows from Lemma 4.2.

(2) The second part is also clear since $x_{1}, x_{2}, \ldots, \widehat{x_{k}}, \ldots, x_{g}$ is a regular sequence on $R$.

Thus Theorem 4.4 enables us to define a complete matrix of grade $g$. With an induction argument, we may call $\bar{T}_{k}$ given in Theorem 4.4 the complete matrix of grade $g-1$ in the following sense.

Definition 4.5. Let $R$ be a commutative ring with identity. Let $g>3$ and $t=\left(\begin{array}{l}g \\ 2\end{array}\right)$ be integers. A $g \times t$ matrix $f=\left(f_{i j}\right)$ over $R$ is said to be complete of grade $g$ if

(1) $f$ has $g$ disjoint pairs $(S, T)$ of a $g \times(g-1)$ submatrix $S$ and a $g \times(t-g+1)$ submatrix $T$;

(2) By removing a row and interchanging columns, each pair $(S, T)$ can be reduced to a pair $(\bar{S}, \bar{T})$, where $\bar{S}$ is a $(g-1) \times(g-1)$ diagonal matrix with 
$\operatorname{det}(\bar{S})=x^{g-1}$ for some $x \in R$, and $\bar{T}$ is the complete matrix of grade $g-1$ with grade $\mathcal{K}_{g-2}(\bar{T})=g-1$.

The following example illustrates Definition 4.5.

Example 4.6. Let $x, y, z, u, w$ be a regular sequence in a Noetherian local ring $R$. Let

$$
f=\left[\begin{array}{cccccccccc}
y & z & u & w & 0 & 0 & 0 & 0 & 0 & 0 \\
-x & 0 & 0 & 0 & z & u & w & 0 & 0 & 0 \\
0 & -x & 0 & 0 & -y & 0 & 0 & u & w & 0 \\
0 & 0 & -x & 0 & 0 & -y & 0 & -z & 0 & w \\
0 & 0 & 0 & -x & 0 & 0 & -y & 0 & -z & -u
\end{array}\right]
$$

The similar argument as in Example 3.4 shows that $f$ satisfies the properties in Proposition 4.3 and the part (2) of Theorem 4.4.

The following theorem is an easy generalization of Theorem 3.5.

Theorem 4.7. Let $g>3$ and $t=\left(\begin{array}{l}g \\ 2\end{array}\right)$ be integers. $A g \times t$ matrix $f=\left(f_{i j}\right)$ over $R$ is a complete matrix of grade $g$.

(1) Every column of $f$ has exactly two nonzero entries.

(2) The number of nonzero rows in each $g \times 2$ submatrix of $f$ is greater than 2.

(3) Each pair $(\bar{S}, \bar{T})$ of matrices given in Definition 4.5 is uniquely determined.

Proof. The proofs are essentially similar with those of Theorem 3.5.

Now we define an ideal $\mathcal{K}_{g-1}(f)$ generated by the entries in the $(g-1) \times(g-1)$ matrices $\bar{S}$ derived from a given complete matrix $f$ of grade $g$ in Theorem 4.7.

Definition 4.8. Let $g>3$ and $t=\left(\begin{array}{l}g \\ 2\end{array}\right)$ be integers. Let $f$ be a $g \times t$ complete matrix of grade $g$. For $i=1,2, \ldots, g$, we let $\bar{S}_{i}$ be a unique $(g-1) \times(g-1)$ diagonal matrix extracted from $f$ in the part (3) of Theorem 4.7 such that $\operatorname{det} \bar{S}_{i}=x_{i}^{g-1}$ is nonzero for some $x_{i} \in R$. We define $\mathcal{K}_{g-1}(f)$ to be the ideal generated by the $x_{i}$ 's, that is,

$$
\mathcal{K}_{g-1}(f)=\left(x_{1}, x_{2}, \ldots, x_{g}\right) .
$$

Let $f=\left(f_{i j}\right)$ be a $g \times t$ complete matrix of grade $g$. It follows from the properties (1) and (2) of Theorem 4.7 that interchanging columns of $f$ transforms $f$ to the following form.

$$
f=\left[\begin{array}{l|l}
h_{1} & 0 \\
\hline d_{1} & h_{2}
\end{array}\right],
$$

where

$$
\begin{aligned}
h_{1} & =\left[\begin{array}{llll}
f_{11} & f_{12} & \cdots & f_{1 g-1}
\end{array}\right], \\
d_{1} & =\operatorname{diag}\left(f_{21}, f_{32}, \ldots, f_{g g-1}\right), \quad h_{2}=\text { a complete matrix of grade } g-1 .
\end{aligned}
$$


By applying the method of (3.5) and (3.6) in the case of a complete matrix of grade 4 to the given $f$, we have

$$
\begin{aligned}
& \mathcal{K}_{g-2}\left(\bar{T}_{1}\right)=\left(\widehat{f}_{21}, f_{11}, f_{12}, \ldots, f_{1 g-1}\right), \text { and } \\
\text { (4.4) } & \mathcal{K}_{g-2}\left(\bar{T}_{i}\right)=\left(f_{21}, f_{11}, f_{12}, \ldots, \widehat{f}_{1 i-1}, f_{1 i}, \ldots, f_{1 g-1}\right) \quad \text { for } i=2,3, \ldots g, \\
& \text { where } \widehat{f}_{1 i} \text { indicates that } f_{1 i} \text { is to be omitted. }
\end{aligned}
$$

Hence

$$
\mathcal{K}_{g-2}\left(\bar{T}_{i}\right) \subseteq \mathcal{K}_{g-1}(f)=\left(f_{21}, f_{11}, f_{12}, \ldots, f_{1 g-1}\right) \text { for each } i .
$$

The following lemma will be used in proving the structure theorem for complete intersections of grade $g>3$.

Lemma 4.9. Let $\mathbf{x}=x_{1}, x_{2}, \ldots, x_{g}$ be a regular sequence on $R$ and $\mathbb{F}$ a minimal free resolution of $R /(\mathbf{x})$. If $\varphi_{2}$ is the second differential map of $\mathbb{F}$, then $\varphi_{2}$ is a complete matrix of grade $g$.

Proof. Let $\mathbb{K}(\mathbf{x})$ be the Koszul complex defined by the regular sequence $\mathbf{x}=$ $x_{1}, x_{2}, \ldots, x_{g}$ and $d_{2}$ the second differential map in $\mathbb{K}(\mathbf{x})$. We have shown in Proposition 4.3 and the part (2) of Theorem 4.4 that $d_{2}$ is a complete matrix of grade $g$. Let $F$ be the free $R$-module with the ordered basis $\left\{e_{1}<e_{2}<\cdots<\right.$ $\left.e_{g}\right\}$. Then $\wedge^{2} F$ is a free $R$-module with the ordered basis $\left\{e_{1} \wedge e_{2}<e_{1} \wedge e_{3}<\right.$ $\left.\cdots<e_{g-1} \wedge e_{g}\right\}$. Let $t=\left(\begin{array}{l}g \\ 2\end{array}\right)$ be an integer. Let

$$
\mathbb{F}: 0 \longrightarrow F_{g} \stackrel{\varphi_{g}}{\longrightarrow} F_{g-1} \stackrel{\varphi_{g-1}}{\longrightarrow} \cdots \stackrel{\varphi_{3}}{\longrightarrow} F_{2} \stackrel{\varphi_{2}}{\longrightarrow} F_{1} \stackrel{\varphi_{1}}{\longrightarrow} R
$$

be the minimal free resolution of $R /(\mathbf{x})$ such that $F_{1}$ and $F_{2}$ are free $R$-modules with the ordered bases $\left\{v_{1}<v_{2}<\cdots<v_{g}\right\}$ and $\left\{w_{1}<w_{2}<\cdots<w_{t}\right\}$, respectively. Then we have a commutative diagram

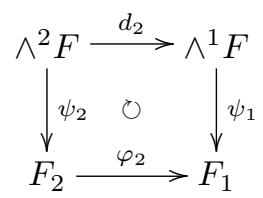

where $\psi_{1}$ and $\psi_{2}$ are order preserving isomorphisms as free $R$-modules. Since $\psi_{1}\left(e_{k}\right)=v_{k}$ for $k=1,2, \ldots, g$ and $\psi_{2}$ maps the $i$ th basis element in $\wedge^{2} F$ to the $i$ th basis element $w_{i}$ in $F_{2}$ for $i=1,2, \ldots, t$, the commutativity implies that $d_{2}$ and $\varphi_{2}$ have the same matrix representation. Thus $\varphi_{2}$ is a complete matrix of grade $g$ since $d_{2}$ is a complete matrix of grade $g$.

Now we can describe a structure theorem for complete intersections of grade $g>3$.

Theorem 4.10. Let $R$ be a Noetherian local ring with maximal ideal $\mathfrak{m}$.

(1) Let $g>3$ be an integer and $t=\left(\begin{array}{l}g \\ 2\end{array}\right)$. Let $F$ and $G$ be free $R$-modules with $\operatorname{rank} F=g$ and $\operatorname{rank} G=t$. Let $f=\left(f_{i j}\right): G \rightarrow F$ be a complete matrix of 
grade $g$ whose image is contained in $\mathfrak{m} F$. With the notation as in Theorem 4.7, we assume that $\mathcal{K}_{g-2}\left(\bar{T}_{i}\right)+\mathcal{K}_{g-2}\left(\bar{T}_{j}\right)$ has grade $g$ for some $i, j(1 \leq i \neq j \leq g)$. Then the ideal $\mathcal{K}_{g-1}(f)$ is a complete intersection of grade $g$.

(2) Let $I=\left(x_{1}, x_{2}, \ldots, x_{g}\right)$ be a complete intersection of grade $g$ and let

$$
\mathbb{F}: 0 \longrightarrow R \stackrel{\varphi_{g}}{\longrightarrow} F_{g-1} \stackrel{\varphi_{g-1}}{\longrightarrow} F_{g-2} \longrightarrow \cdots \longrightarrow F_{2} \stackrel{\varphi_{2}}{\longrightarrow} F_{1} \stackrel{\varphi_{1}}{\longrightarrow} R
$$

be the minimal free resolution of $R / I$. Then $\varphi_{2}$ and the transpose of $\varphi_{g-1}$ satisfy (1).

Proof. (1) We showed in Theorem 3.7 that the first part of the theorem is true for the case of $g=4$. Let $f=\left(f_{i j}\right)$ be a $g \times t$ complete matrix of grade $g$. As shown in Proposition 4.3, interchanging columns of $f$ transforms $f$ to the form of (4.3). So we may assume that $f$ has the form of (4.3). Then we have

$$
\mathcal{K}_{g-1}(f)=\left(f_{21}, f_{11}, f_{12}, \ldots, f_{1 g-1}\right) .
$$

Since $\mathcal{K}_{g-2}\left(\bar{T}_{i}\right)+\mathcal{K}_{g-2}\left(\bar{T}_{j}\right)$ has grade $g$ for some $i, j(i \neq j)$, it follows from (4.4) and (4.5) that $\mathcal{K}_{g-1}(f)$ is a complete intersection of grade $g$. Let $\mathbf{x}=f_{21}, f_{11}, f_{12}, \ldots, f_{1 g-1}$. Then $\mathbf{y}_{1}=\widehat{f}_{21}, f_{11}, f_{12}, \ldots, f_{1 g-1}$ and each $\mathbf{y}_{i}=$ $f_{21}, f_{11}, f_{12}, \ldots, \widehat{f}_{1 i-1}, \ldots, f_{1 g-1}$ for $i>1$ are regular sequences. From (4.4), $f_{21}$ is regular on $R / \mathcal{K}_{g-2}\left(\bar{T}_{1}\right)$, and $f_{1 i-1}$ is regular on $R / \mathcal{K}_{g-2}\left(\bar{T}_{i}\right)$ for $i>1$. Let $\mathbb{G}_{i}$ be a complex of free R-modules such that

$$
\mathbb{G}_{1}: 0 \longrightarrow R \stackrel{f_{21}}{\longrightarrow} R,
$$

and for $i>1$,

$$
\mathbb{G}_{i}: 0 \longrightarrow R \stackrel{f_{1 i-1}}{\longrightarrow} R .
$$

Then by the part (1) of Lemma 4.1, $\mathbb{G}_{i} \otimes \mathbb{K}\left(\mathbf{y}_{i}\right)$ is a minimal free resolution of $R / \mathcal{K}_{g-1}(f)$.

(2) We showed in Theorem 3.7 that the part (2) holds for the case of $g=$ 4. Let $I=\left(x_{1}, x_{2}, \ldots, x_{g}\right)$ be a complete intersection of grade $g$ and $I^{\prime}=$ $\left(x_{2}, x_{3}, \ldots, x_{g}\right)$ be a complete intersection of grade $g-1$. The same argument as in the proof of the part (2) of Theorem 3.7 says that $\varphi_{2}$ in (4.6) is of the form

$$
\varphi_{2}=\left[\begin{array}{c|c}
\tilde{\varphi}_{1} & 0 \\
\hline \tilde{d} & \tilde{\varphi}_{2}
\end{array}\right],
$$

where

$$
\tilde{\varphi}_{1}=\left[\begin{array}{llll}
-x_{2} & -x_{3} & \cdots & -x_{g}
\end{array}\right], \quad \tilde{d}=\operatorname{diag}\left(x_{1}, x_{1}, \ldots, x_{1}\right),
$$

and $\tilde{\varphi}_{2}$ is the second differential map of the minimal free resolution of $R / I^{\prime}$. Lemma 4.9 says that $\tilde{\varphi}_{2}$ is a complete matrix of grade $g-1$. Since $x_{1}, x_{2}, \ldots, x_{g}$ is a regular sequence on $R$, Lemma 4.9 implies that $\varphi_{2}$ is a complete matrix of grade $g$. We observe that every row of $\varphi_{2}$ consists of $g-1$ nonzero entries and 
$t-g+1$ zero entries. The similar argument as in the proof of Proposition 4.3 gives us the following: Let $\left(\bar{S}_{i}, \bar{T}_{i}\right)$ be a pair of a $(g-1) \times(g-1)$ diagonal matrix and a $(g-1) \times(t-g+1)$ complete matrix of grade $g-1$. Then for $i=1,2, \ldots, g$,

$$
\operatorname{det} \bar{S}_{i}= \pm x_{i}^{g-1}, \mathcal{K}_{g-2}\left(\bar{T}_{i}\right)=\left(x_{1}, x_{2}, \ldots, \widehat{x}_{i}, x_{i+1}, \ldots, x_{g}\right) .
$$

So we have

$$
\begin{aligned}
& \mathcal{K}_{g-1}\left(\varphi_{2}\right)=\left(x_{1}, x_{2}, \ldots, x_{g}\right) \text {, and } \mathcal{K}_{g-2}\left(\bar{T}_{i}\right)+\mathcal{K}_{g-2}\left(\bar{T}_{j}\right)=\mathcal{K}_{g-1}\left(\varphi_{2}\right) \\
& \text { for some } i \neq j .
\end{aligned}
$$

We know that each $\mathcal{K}_{g-2}\left(\bar{T}_{i}\right)$ has grade $g-1$, and $\mathcal{K}_{g-1}\left(\varphi_{2}\right)$ is a complete intersection of grade $g$. Hence $\varphi_{2}$ satisfies the part (1) of Theorem 4.10. Since every complete intersection is Gorenstein, $\mathbb{F} \cong \mathbb{F}^{*}$ as complexes. So $\mathbb{F}^{*}$ is the minimal free resolution of $R / I$. The same argument as in the proof of the part (2) of Theorem 3.7 for $\mathbb{K}(\mathbf{x})$ and $\mathbb{F}^{*}$ gives us the proof that the transpose of $\varphi_{g-1}$ is a complete matrix of grade $g$.

It should be noticed that Theorem 3.7 is just the special case of $g=4$ in Theorem 4.10. The following example illustrates how Theorem 4.10 works.

Example 4.11. Let $\mathbb{C}$ be the field of the complex numbers and $R$ the formal power series ring $\mathbb{C}\left[\left[x_{i j}, y, z, w, u \mid 1 \leq i, j \leq 3\right]\right]$ over $\mathbb{C}$ with indeterminates $x_{i j}, y, z, w, u$. Consider a $3 \times 3$ matrix $X$ and a $3 \times 3$ alternating matrix $Y$

$$
X=\left[\begin{array}{lll}
x_{11} & x_{12} & x_{13} \\
x_{21} & x_{22} & x_{23} \\
x_{31} & x_{32} & x_{33}
\end{array}\right], \quad Y=\left[\begin{array}{ccc}
0 & w & z \\
-w & 0 & y \\
-z & -y & 0
\end{array}\right]
$$

Define

$$
Z_{1}=\sum_{i=1}^{3} Y_{i} x_{i 1}, \quad Z_{2}=\sum_{i=1}^{3} Y_{i} x_{i 2}, \quad Z_{3}=\sum_{i=1}^{3} Y_{i} x_{i 3}, \quad v=\operatorname{det} X .
$$

Then $I=\left(Z_{1}, Z_{2}, Z_{3}, v\right)$ is an almost complete intersection of grade 3 of type $3[1,5]$. Assume that $\mathbf{z}=Z_{1}, Z_{2}, Z_{3}$ is a regular sequence on $R$. Then

$$
J=(\mathbf{z}): I=\left(Y_{1}, Y_{2}, Y_{3}\right)=(y, z, w) .
$$

Since $v$ is not contained in the ideal $J, I \cap J=(\mathbf{z})$. Hence $I$ is geometrically linked to $J$ by a regular sequence $\mathbf{z}$. Thus by Theorem $2.3, K=I+J=$ $(y, z, w, v)$ is a complete intersection of grade 4 . So $\mathbf{x}=y, z, w, v$ is a regular sequence on $R$. We may assume that $u$ is a regular element on $R / K$. Thus $H=(y, z, w, v, u)$ is a complete intersection of grade 5 . Let

$$
\mathbb{K}(u): 0 \longrightarrow R \stackrel{u}{\longrightarrow} R
$$

be a complex of free $R$-modules and $R$-maps. Then $\mathbb{H}=\mathbb{K}(u) \otimes \mathbb{K}(\mathbf{x})$ described as in the part (2) of Lemma 4.1 is the minimal free resolution of $R / H$. Let $\phi_{2}$ be the second differential map in $\mathbb{H}$. Since $y, z, w, v, u$ is a regular sequence 
on $R$, by Lemma $4.9, \phi_{2}$ is a complete matrix of grade 5 . It is easy to show that $\mathcal{K}_{4}\left(\phi_{2}\right)=(u, y, z, w, v)$ is a complete intersection of grade 5. Moreover, we let $\bar{T}_{i}$ be a $4 \times 6$ complete matrix of grade 4 with the same notation, $\bar{T}_{i}$ in Definition 4.5. Then we have

$$
\begin{array}{ll}
\mathcal{K}_{3}\left(\bar{T}_{1}\right)=(y, z, w, v), & \mathcal{K}_{3}\left(\bar{T}_{2}\right)=(u, z, w, v), \quad \mathcal{K}_{3}\left(\bar{T}_{3}\right)=(u, y, w, v), \\
\mathcal{K}_{3}\left(\bar{T}_{4}\right)=(u, y, z, v), & \mathcal{K}_{3}\left(\bar{T}_{5}\right)=(u, y, z, w) .
\end{array}
$$

Hence $\mathcal{K}_{3}\left(\bar{T}_{i}\right)+\mathcal{K}_{3}\left(\bar{T}_{j}\right)=\mathcal{K}_{4}\left(\phi_{2}\right)$ for some $i \neq j$. This illustrates the Theorem 4.10 .

\section{References}

[1] A. Brown, A structure theorem for a class of grade three perfect ideals, J. Algebra 105 (1987), no. 2, 308-327.

[2] D. A. Buchsbaum and D. Eisenbud, Algebra structures for finite free resolutions, and some structure theorems for ideals of codimension 3, Amer. J. Math. 99 (1977), no. 3, 447-485.

[3] L. Burch, On ideals of finite homological dimension in local rings, Proc. Cam. Phil. Soc. 64 (1968), 941-948.

[4] O.-J. Kang and H. J. Ko, The structure theorem for complete intersections of grade 4, Algebra Colloq. 12 (2005), no. 2, 181-197.

[5] A. Kustin and M. Miller, Structure theory for a class of grade four Gorenstein ideals, Trans. Amer. Math. Soc. 270 (1982), no. 1, 287-307.

[6] C. Peskine and L. Szpiro, Liaison des variétés algébriques. I, Invent. Math. 26 (1974), 271-302.

[7] R. Sánchez, A structure theorem for type 3, grade 3 perfect ideals, J. Algebra 123 (1989), no. $2,263-288$.

Eun JEONG ChOI

University College

YONSEI UNIVERSITY

SeOUl 120-749, KoreA

E-mail address: eunjchoi@yonsei.ac.kr

OH-JIN KANG

Department of Mathematics

Science College

UNIVERSITY OF INCHEON

INCHEON 402-749, KorEA

E-mail address: ohkang@incheon.ac.kr

HYOUNG J. KO

DePARTMENT OF MATHEMATICS

YONSEI UNIVERSITY

SeOUl 120-749, KoreA

E-mail address: hjko@yonsei.ac.kr 